\title{
Results of the PARAMEDIC-2 trial and how they relate to resuscitation after cardiac surgery
}

Joel Dunning, MD, $\mathrm{PhD}$, and Jason Trevis, MD

The PARAMEDIC- 2 trial $^{1}$ is a landmark trial in the field of resuscitation that has gone a long way toward answering a question that many clinicians not involved in resuscitation research may be surprised to find out exists at all. The question is whether epinephrine is useful for resuscitation of patients who suffer a cardiac arrest. The reason that I say that this is a surprise to many is that most clinicians assume that this cornerstone of resuscitation must have a rock-solid foundation. As junior doctors, we all go on resuscitation courses, and many of us will have spent some years on hospital resuscitation teams, and we have always learned the exact timing of epinephrine administration for those experiencing cardiac arrest. It is one of the first things we do in an arrest after external chest compressions have been commenced, airway and breathing have been secured, and we have considered defibrillation. The next most important task has always been the quick administration of epinephrine once we have gained intravenous (IV) access.

So shaking my "belief" in epinephrine was certainly a surprise to me in 2010, when I was at the International Liaison Committee on Resuscitation (ILCOR) conference in Dallas, which they hold every 5 years to fully update the knowledge base of resuscitation in all specialties. This conference brings together the 7 world resuscitation councils, which work together to agree on the evidence base in every area before writing their own resuscitation guidelines in their 7 regions. My belief was shaken by a full literature review on epinephrine ${ }^{2}$ as well as a large randomized controlled trial (RCT) of ambulances in Norway published by Olasveengen and colleagues ${ }^{3}$ which were randomized to 2 groups: cardiopulmonary resuscitation (CPR) and defibrillation with IV cannulation and usual resuscitation medications versus CPR and defibrillation alone. This RCT found an adverse survival to hospital discharge in the first group, which was believed to be due

From the Department of Thoracic Surgery, James Cook University Hospital, Middlesbrough, United Kingdom.

Received for publication May 9, 2019; revisions received Jan 28, 2020; accepted for publication Feb 12, 2020; available ahead of print March 30, 2020.

Address for reprints: Joel Dunning, MD, PhD, Department of Thoracic Surgery, James Cook University Hospital, Marton Rd, Middlesbrough TS4 3BW, United Kingdom (E-mail: Joeldunning@ doctors.org.uk)

J Thorac Cardiovasc Surg 2020;160:1519-22 0022-5223/\$36.00

Copyright (c) 2020 Published by Elsevier Inc. on behalf of The American Association for Thoracic Surgery

https://doi.org/10.1016/j.jtcvs.2020.02.050

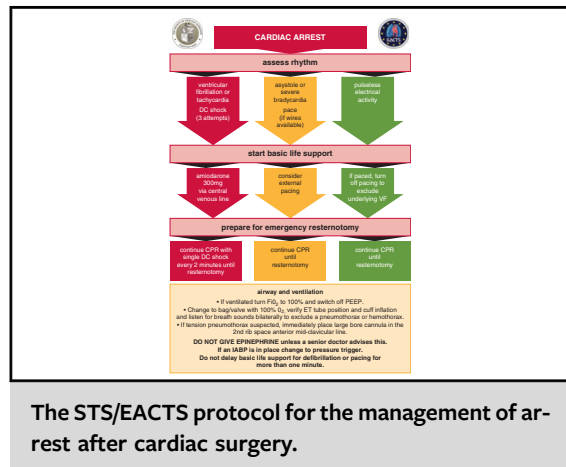

\begin{abstract}
CENTRAL MESSAGE
The PARAMEDIC-2 Trial is a randomized controlled trial of 8014

patients who experienced an

out-of-hospital cardiac arrest.

We discuss its results and their

application to the Society of

Thoracic Surgeons protocol for

the management of patients who

arrest after cardiac surgery.

This Invited Expert Opinion provides a perspective on the following paper: N Engl J Med. 2018; 379:711-721. https:/doi.org/10.1056/NEJMoa1806842.
\end{abstract}

See Commentaries on pages 1523 and 1524 .

to the ineffectiveness of the drugs and also the reduction of CPR to cannulate and administer the drugs. The aforementioned literature review as well a more recent meta-analysis ${ }^{4}$ demonstrating no benefit of epinephrine in cardiac arrest led the International Liaison Committee on Resuscitation to report that

despite the widespread use of epinephrine during resuscitation, and several studies involving vasopressin, there is no placebo-controlled study that shows that the routine use of any vasopressor at any stage during human cardiac arrest increases survival to hospital discharge. Current evidence is insufficient to support or refute the routine use of 
any particular drug or sequence of drugs. Despite the lack of human data, the use of adrenaline is still recommended, based largely on animal data.

This is the background in which the PARAMEDIC-2 study was performed. This is truly a landmark trial in many respects. It successfully randomized 8014 patients in an arrest situation across 5 ambulance services in the United Kingdom. You may ask how patients can possibly be randomized in this situation, but this was elegantly dealt with by deferring consent until the emergency had passed. Clearly, patients will either have had or not have had treatment by this point, but the consent was provided by the patient (or much more often by their legal representative) mainly to collect and store their data and follow up for outcomes. Only 6 patients/representatives declined consent and thus were withdrawn. Randomization was done by the opening of a trial pack after CPR and defibrillation were unsuccessful, which contained syringes of either $1 \mathrm{mg}$ of epinephrine for use every 3 to 5 minutes or exactly identical syringes containing $0.9 \%$ saline. The average time of ambulance arrival was 6.6 minutes, and the mean time to trial drug administration was 13 minutes after arrival.

In terms of results, there was a large increase in the number of patients who had a return of spontaneous circulation with epinephrine ( $36 \%$ vs $11 \%$ ) and the number who were transferred to the hospital $(50 \%$ vs $30 \%)$. The primary outcome measure was survival at 30 days, which was $3.2 \%$ in the epinephrine group and $2.4 \%$ in the placebo group, which were significant, but the number of patients with severe neurologic impairment was $31 \%$ in the epinephrine group versus $18 \%$ in the control group, and thus the trial was negative in terms of survival with favorable neurologic outcome ( $2.2 \%$ vs $1.9 \%)$. Thus, the triallists concluded that epinephrine significantly improved the chance of achieving return of spontaneous circulation and the patient to the hospital, but that this led only to a higher proportion of patients surviving with severe neurologic disability.

They also made some other very interesting observations. The number needed to treat of early recognition of the arrest was 11 , that of bystander CPR was 15 , and that with early defibrillation was only 5 . In comparison, the number needed to treat with epinephrine to achieve survival with any neurology was 112 .

So for the world resuscitation community there is now a dilemma regarding whether epinephrine remains in the algorithms, given the knowledge that lives can occasionally be saved, but these lives may well be low-quality lives with severe neurology to cope with. Much work has been done with patient advisory groups, and the strong message is that patients strongly favor survival with favorable neurology over survival alone, ${ }^{5}$ and thus it is certainly possible that epinephrine may well find itself removed from the general resuscitation algorithm in the same way that atropine was removed after publication of the RCT of Olasveengen and colleagues ${ }^{2}$ mentioned above.

So how does this affect us in resuscitation after cardiac surgery? The management of our patients is directed by The Society of Thoracic Surgeons expert consensus for the resuscitation of patients who arrest after cardiac surgery published in $2017,{ }^{6}$ a comprehensive set of protocols adapted specifically for our unique patients (Figure 1). It acknowledges that the standard adult advanced life support algorithm is not suitable for use in our cardiac intensive care units. Departures from the standard protocol include not starting external chest compressions until defibrillation or pacing has been attempted. Elements include attenuating any external chest compressions to approximately $60 \mathrm{~mm}$ $\mathrm{Hg}$, performing a standard endotracheal tube check, excluding tension pneumothorax, and stopping all infusions, but the main focus is on achieving an emergency resternotomy within a time frame of 5 minutes to relieve tamponade, identify any major bleeding points, and commence internal cardiac massage. This rapid resternotomy is key to the now-proven survival benefits of using this protocol and the STS-recommended training in teams, and the training program set up by the Association of Physician Assistants in Cardiovascular Surgery (www.csu-als. com) is recommended to achieve this difficult task for all units performing cardiac surgery.

One key recommendation from the STS paper is also to not administer epinephrine in a cardiac arrest after cardiac surgery. In fact, we have been recommending that epinephrine not be given to patients who arrest after cardiac surgery since $2009,{ }^{7}$ based on our literature review specific to patients after cardiac surgery. ${ }^{8}$ We considered 2 issues-first, whether epinephrine would benefit the patient, and second, whether epinephrine could cause harm. The Adult ALS algorithm states that in an asystolic arrest, epinephrine should be administered as soon as IV access is obtained. However, consider a patient who arrests 3 hours after a double-valve replacement due to loss of capture of their temporary pacemaker; imagine the harm that could be done if someone gave $1 \mathrm{mg}$ of epinephrine and then increased the amplitude of the temporary pacemaker to immediately gain capture. In fact, we found a case of a patient who arrested after an aortic valve replacement and coronary artery bypass grafting who was given epinephrine but then had a tension pneumothorax identified. ${ }^{9}$ This was immediately resolved, but the resulting extreme hypertension due to the epinephrine caused massive blood loss, resulting in arrest and emergency resternotomy to repair the aortotomy suture line. Therefore, 


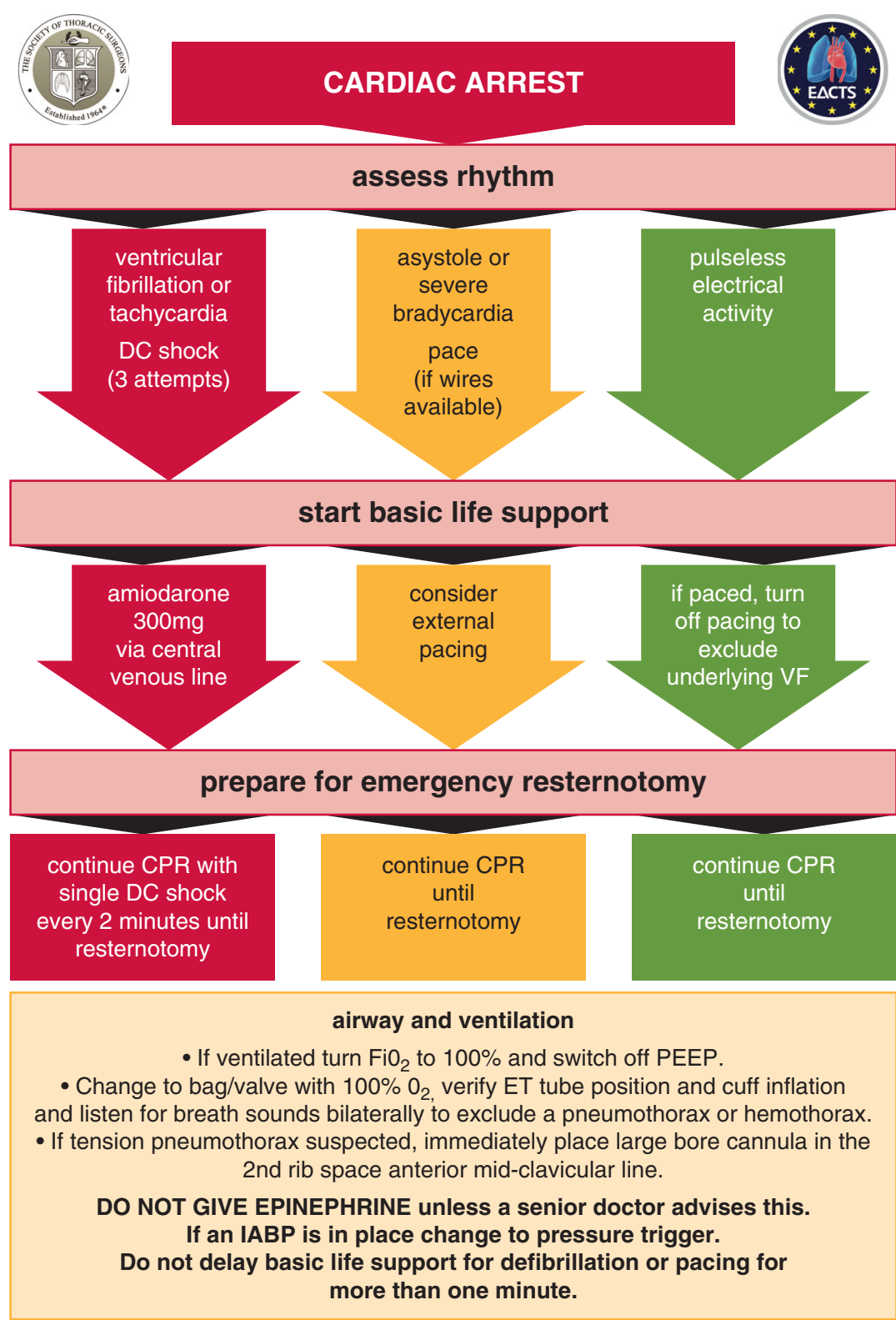

FIGURE 1. The STS/EACTS protocol for resuscitation after cardiac surgery. $V F$, ventricular fibrillation; $C P R$, cardiopulmonary resuscitation; $D C$, direct current; PEEP, positive end-expiratory pressure; IABP, intra-aortic balloon pump.

we strongly feel that unless all immediately reversible causes are excluded, epinephrine should not be given. One of those immediately reversible causes is tamponade, and thus it certainly should not be given before an emergency resternotomy.

We are confident that epinephrine can potentially cause harm in patients who arrest after cardiac surgery, but might it be of benefit. The PARAMEDIC-2 trial strongly confirms our belief that epinephrine will be of no benefit to our patients. In the PARAMEDIC-2 study, the drug was administered an average 19 minutes after the arrest was identified, and the number needed to treat was 112 to increase the chance of having a survivor to hospital discharge, but potentially with worse neurology. The STS protocol recommends emergency resternotomy within 5 minutes of an arrest, after all potentially reversible causes have been identified, and thus at 19 minutes when this study looked at the use of epinephrine, we would have performed 14 minutes of internal cardiac compressions, which has been shown to be far superior to external compressions, ${ }^{10}$ and hopefully 
would be looking to be going back on cardiac bypass if cardiac recovery did not occur.

We do not exclude the use of epinephrine in the periarrest situation in a patient who perhaps might benefit from 50 to $300 \mu \mathrm{g}$ of epinephrine and even possibly some external chest compressions to get the drug around the patient, but we reserve this use for expert clinicians, who themselves would be very unlikely to want to use it at its full 1-mg dose.

Therefore, we recommend following the PARAMEDIC-2 recommendations in conjunction with the STS expert consensus for the resuscitation of patients who arrest after cardiac surgery. We feel that these lend further support to our comprehensive protocol that has now been shown worldwide to save lives in patients who arrest after cardiac surgery.

\section{Conflict of Interest Statement}

Authors have nothing to disclose with regard to commercial support.

\section{References}

1. Perkins GD, Ji C, Deakin CD, Quinn T, Phil M, Nolan JP, et al. A randomized trial of epinephrine in out-of-hospital cardiac arrest. N Engl J Med. 2018;379:711-21.
2. Long G, Little CM, Paradis NA. W83E: epinephrine (at either standard doses or higher doses) is a safe and effective adjunct to defibrillation in cardiac arrests due to ventricular fibrillation. Circulation. 2005;112(Suppl):B1-14.

3. Olasveengen TM, Wik L, Sunde K, Steen PA. Outcome when adrenaline (epinephrine) was actually given vs. not given — post hoc analysis of a randomized clinical trial. Resuscitation. 2012;83:327-32.

4. Lin S, Callaway CW, Shah PS, Wagner JD, Beyene J, Ziegler CP, et al. Adrenaline for out-of-hospital cardiac arrest resuscitation: a systematic review and meta-analysis of randomized controlled trials. Resuscitation. 2014;85: 732-40.

5. Haywood K, Whitehead L, Nadkarni VM, Achana F, Beesems S, Böttiger BW, et al. COSCA (Core Outcome Set for Cardiac Arrest) in adults: an advisory statement from the International Liaison Committee on Resuscitation. Resuscitation. 2018;127:147-63.

6. Society of Thoracic Surgeons Task Force on Resuscitation After Cardiac Surgery. The Society of Thoracic Surgeons expert consensus for the resuscitation of patients who arrest after cardiac surgery. Ann Thorac Surg. 2017; 103:1005-20.

7. Dunning J, Fabbri A, Kolh PH, Levine A, Lockowandt U, Mackay J, et al. Guideline for resuscitation in cardiac arrest after cardiac surgery. Eur J Cardiothorac Surg. 2009;36:3-28.

8. Tsagkataki M, Levine A, Strang T, Dunning J. Should adrenaline be routinely used by the resuscitation team if a patient suffers a cardiac arrest shortly after cardiac surgery? Interact Cardiovasc Thorac Surg. 2008;7: 457-62.

9. Webb ST. Caution in the administration of adrenaline in cardiac arrest following cardiac surgery. Resuscitation. 2008;78:101.

10. Raman J, Saldanha RF, Branch JM, Esmore DS, Spratt PM, Farnsworth AE, et al. Open cardiac compression in the postoperative cardiac intensive care unit. Anaesth Intensive Care. 1989;17:129-35. 